\title{
Aharonov-Bohm effect in a helical ring with long-range hopping: Effects of Rashba spin-orbit interaction and disorder
}

\author{
Paramita Dutta, ${ }^{1, *}$ Arijit Saha, ${ }^{1, \dagger}$ and A. M. Jayannavar ${ }^{1, \ddagger}$ \\ ${ }^{1}$ Institute of Physics, Sachivalaya Marg, Bhubaneswar-751005, India
}

\begin{abstract}
We study Aharonov-Bohm effect in a two-terminal helical ring with long-range hopping in presence of Rashba spin-orbit interaction. We explore how the spin polarization behavior changes depending on the applied magnetic flux and the incoming electron energy. The most interesting feature that we articulate in this system is that zero-energy crossings appear in the energy spectra at $\Phi=0$ and also at integer multiples of half-flux quantum values $\left(n \Phi_{0} / 2, n\right.$ being an integer) of the applied magnetic flux. We investigate the transport properties of the ring using Green's function formalism and find that the zero energy transmission peaks corresponding to those zero energy crossings vanish in presence of Rashba spin-orbit interaction. We also incorporate static random disorder in our system and show that the zero energy crossings and transmission peaks are not immune to disorder even in absence of Rashba spin-orbit interaction. The latter prevents the possibility of behaving these helical states in the ring like topological insulator edge states.
\end{abstract}

PACS numbers: 73.23.-b,72.25.-b,71.70.Ej

\section{INTRODUCTION}

During the last two decades manipulating and controlling electronic spin, one of the most fundamental degrees of freedom, has introduced a new paradigm in the field of quantum condensed matter physics especially in spintronics $^{1,2}$. It has drawn interests of the scientific community due to the prospects of application in modern quantum devices. Till date, various spintronic phenomena like spin-switching, spin filtering, spin-splittering etc. have been proposed using ferromagnetic leads, external magnetic field and so on ${ }^{3,4}$. Internal properties of the system have also been utilized for maneuvering the spin current in a precise way ${ }^{5}$. Internal properties like spinorbit interaction (SOI), especially Rashba spin-orbit interaction (RSOI) ${ }^{6,7}$ which arises due to the structural inversion asymmetry yields an alternative way of spin manipulation $^{8}$. The tunability of RSOI strength by external gate voltage placed in vicinity of the sample offers an additional degree of freedom in this context ${ }^{9}$.

A large number of spintronic devices have been proposed on the basis of quantum interferometers among which the simplest one is the ring geometry ${ }^{10}$. The quasi-one-dimensional Aharonov-Bohm rings are elegant test-beds for exploring the quantum coherence phenomena ${ }^{11-17}$. The simplicity of its topological geometry has drawn attention of researchers due to their potential application in various nano-electronic devices. Research activity on these ring structures have been boosted after the recent advancement of nano-technology which has made it possible to fabricate metallic as well as semi-conductor rings in desired way ${ }^{18-22}$. To study the interference effect in such a ring geometry, Aharonov-Bohm (AB) flux is a key ingredient as it affects only the phases of the electronic wave functions ${ }^{23}$. In ordinary mesoscopic ring $\mathrm{AB}$ effect deals with the charge of the electrons keeping the spin degeneracy intact. Therefore, breaking the spin degeneracy can be an interesting aspect ${ }^{24}$. This in- vokes one to think about the spin $A B$ effect where the spin manipulation can be done by $\mathrm{AB}$ flux utilizing the spin-dependent phase factors introduced by SOI. This is a non-local phenomenon ${ }^{25}$.

Indeed, this type of spin manipulation by magnetic flux can be done by using the helical edge states which is observed in topologically non-trivial new state of mat$\operatorname{ter}^{26,27}$. At the boundary of a two-dimensional (2D) topological insulator (TI), a pair of counter-propagating states with two opposite spins and protected by timereversal symmetry appear ${ }^{28,29}$. These $1 \mathrm{D}$ gapless helical edge states are robust to static disorder resulting in the absence of back-scattering, and they cannot be perturbed as long as time-reversal symmetry is preserved. They were theoretically predicted in quantum spin Hall insulators $^{30,31}$. Later, Bernevig et al. $^{32}$ have investigated the band structures of $\mathrm{HgTe} / \mathrm{CdTe}$ heterostructrues and shown a transition from topologically trivial to non-trivial state of matter by means of change in width of the heterostructure. However, the existence of these $1 \mathrm{D}$ edge states was experimentally realized in HgTe/CdTe heterostructrues by König et al. ${ }^{33}$. This path-breaking observation stimulated the research in this direction both theoretically ${ }^{34-37}$ and experimentally ${ }^{38,39}$. In order to understand the behavior of these helical edge states characterized by linear dispersion, various models have been proposed in literature like, Kane Mele model $^{30,40}$, Bernevig-Hughes-Zhang (BHZ) model ${ }^{32}$ etc. Parallel to the continuum model, lattice models have also been proposed to describe this state of matter being topologically distinct from all other known states of matter ${ }^{41}$. Although it is difficult to map them in a lattice model since one faces the problem of Fermion doubling ${ }^{42}$.

In a very recent paper Masuda et al. have suggested a model where one can get rid of the Fermion doubling problem $^{43}$ in a lattice model. They have considered a $1 \mathrm{D}$ ring with long-range hopping that stimulates the $1 \mathrm{D}$ edge states of a $2 \mathrm{D}$ topological insulator neglecting the lateral distribution of wave function in real system. This 
long-range hopping model was actually introduced by Gebhard and Ruckenstein ${ }^{44}$. Nevertheless, their purpose was to explore Mott-Hubbard metal-insulator transition in this model. While, Masuda et al. have modified their model to obtain a 1D interferometer with helical spin current and predicted a way to generate spin polarized current by injecting completely spin unpolarized electrons.

This type of 1D model with long-range hopping have already been used earlier to describe several physical systems like, cold atoms, ion traps etc ${ }^{45-53}$ also in biomolecules ${ }^{54-56}$. Very recently, Celardo et al. have studied shielding and localization phenomena in a paradigmatic model of $1 \mathrm{D}$ ring with long-range hopping ${ }^{57}$. Whereas, manifestation of $1 \mathrm{D}$ edge states by a simple ring geometry is a new one. However, Masuda et al. ${ }^{43}$ have not considered the role of Rashba spin-orbit interaction which plays a crucial role in modeling the quantum spin Hall states. In addition to this, the phenomenon of quantum interference can be more interesting in presence of AB flux. Now, the effect of AB flux in 2D TI ring is already well-explored phenomenon ${ }^{58,59}$. Most of the previous works were done in order to study the circulating current within the closed boundary. A very few studies have been made to investigate the transmission or the conductance of electrons in open systems comprising of 1D helical states being attached to leads in presence of magnetic flux. As an example, Chu et al. have shown periodic oscillations in magnetoconductance leading to a possibility of giant magnetoresistance that may be utilized in practical application ${ }^{60}$.

Motivated by the above mentioned facts, we explore the spin-dependent transport phenomena in a $1 \mathrm{D}$ ring with long-range hopping in presence of $\mathrm{AB}$ flux and Rashba SOI. We predict the existence of zero-energy crossings at zero and integer multiples of half-flux quantum values of the applied magnetic flux. We attach two 1D semi-infinite leads to the ring and studied the transport properties using Green's function formalism ${ }^{61}$. In the transmission spectra we notice the zero-energy peaks corresponding to zero-energy states in the energy spectra for the same flux values. However, the zero-energy crossing as well as the associated zero-energy peaks corresponding to the zero energy states no longer exist in presence of RSOI. Instead, a gap appears in the energy and corresponding transmission spectra around the zeroenergy value. We also incorporate static disorder into the system and examine whether zero-energy crossings are immune to disorder which is one of the essential features of $1 \mathrm{D}$ helical edge states in $2 \mathrm{D}$ topological insulators. We observe that the zero-energy states and the peaks get affected by the presence of non-magnetic impurity even in absence of RSOI. They are not robust to disorder. Hence, these 1D helical states are sensitive to both RSOI and disorder.

The remainder of this paper is organized as follows. We describe our model and Hamiltonian in Sec. II and the Green's function formalism in Sec. III in order to calculate the transmission probability of electrons. In
Sec. IV we discuss our numerical results which include the effect of AB flux, RSOI and disorder. Finally, we summarize and conclude in Sec. V.

\section{MODEL}

In Fig. 1 we present our geometry in which a onedimensional (1D) ring is attached to two semi-infinite 1D leads namely, lead-1 and lead-2. The ring has $N$ number of atomic sites periodically arranged with the lattice spacing $a$. We consider $N$ as odd number and it is necessary for this model in order to get the linear dispersion relation as mentioned in Ref. 43. Two circular arrows of magenta and blue colors indicate the direction of current flow along clockwise and anti-clockwise directions corresponding to two opposite spins, respectively. The ring is penetrated by an Aharonov-Bohm (AB) flux $\Phi$ along its axis (upward direction). We describe our model by tight-binding (TB) Hamiltonian within non-interacting electron picture.

The Hamiltonian for the entire system, ring with two side-attached leads, can be partitioned as,

$$
\boldsymbol{H}=\left(\begin{array}{ccc}
\boldsymbol{H}_{\mathrm{L}_{1}} & \boldsymbol{H}_{\mathrm{L}_{1} \mathrm{R}} & 0 \\
\boldsymbol{H}_{\mathrm{L}_{1} \mathrm{R}}^{\dagger} & \boldsymbol{H}_{\mathrm{R}} & \boldsymbol{H}_{\mathrm{RL}_{2}} \\
0 & \boldsymbol{H}_{\mathrm{RL}_{2}}^{\dagger} & \boldsymbol{H}_{\mathrm{L}_{2}}
\end{array}\right)
$$

where, $\boldsymbol{H}_{\mathrm{R}}, \boldsymbol{H}_{\mathrm{L}_{1}}$ and $\boldsymbol{H}_{\mathrm{L}_{2}}$ describe the Hamiltonian for the ring, lead-1 and lead-2, respectively. $\boldsymbol{H}_{\mathrm{L}_{1(2)} \mathrm{R}}$ represents the coupling between ring and lead-1(2).

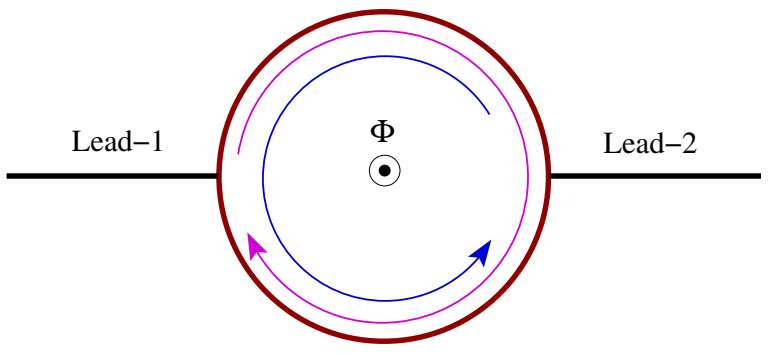

FIG. 1. (Color online) Schematic diagram of 1D helical ring attached to two 1D semi-infinite leads viz. lead-1 and lead-2. Aharonov-Bohm flux $\Phi$ is applied along the axis of the ring (upward direction). Magenta and blue circular arrows represent the two counter-propagating current flows with opposite spins within the ring.

The Hamiltonian for the $1 \mathrm{D}$ ring can be written in Wannier basis as,

$$
\boldsymbol{H}_{\mathrm{R}}=\sum_{m} \boldsymbol{\epsilon}_{m} \mathbf{c}_{m}^{\dagger} \mathbf{c}_{m}+\sum_{m \neq n}\left(\mathbf{t}_{m, n} \mathbf{c}_{m}^{\dagger} \mathbf{c}_{n} e^{i \Theta_{m, n}}+\text { h.c. }\right)
$$

where,

$$
\mathbf{c}_{m}^{\dagger}=\left(\begin{array}{ll}
\mathbf{c}_{m, \uparrow}^{\dagger} & \mathbf{c}_{m, \downarrow}^{\dagger}
\end{array}\right), \mathbf{c}_{m}=\left(\begin{array}{l}
\mathbf{c}_{m, \uparrow} \\
\mathbf{c}_{m, \downarrow}
\end{array}\right)
$$


Here, $m, n$ are the site indices and $\uparrow(\downarrow)$ refers to spin index according to $S_{z}+$ and $S_{z}-$, respectively with the $z$ axis along the direction perpendicular to the plane of the ring. $\mathbf{c}_{m \sigma}^{\dagger}\left(\mathbf{c}_{m \sigma}\right)$ is the creation (annihilation) operator at $m$-th site for an electron with spin $\sigma$. The on-site energy matrix is

$$
\boldsymbol{\epsilon}_{m}=\epsilon\left(\begin{array}{ll}
1 & 0 \\
0 & 1
\end{array}\right)
$$

where $\epsilon$ is the on-site energy parameter set to zero. $\mathbf{t}_{m, n}$ is the long-range hopping integral between $m$-th and $n$-th sites. It has two parts corresponding to the bare hopping integral (i.e. hopping due to bonding) and the hopping due to Rashba spin-orbit coupling. We write it as,

$$
\mathbf{t}_{m, n}=\mathbf{t}_{m, n}^{0}+\mathbf{t}_{m, n}^{\prime}
$$

where the bare hopping part is given by,

$$
\mathbf{t}_{m, n}^{0}=t_{m, n}^{l}\left(\begin{array}{cc}
1 & 0 \\
0 & -1
\end{array}\right) \text {. }
$$

In contrast to an ordinary hopping parameter inside the ring we consider an imaginary term for the long-range hopping integral expressed $\operatorname{as}^{43}$,

$$
t_{m, n}^{l}=\frac{i t(-1)^{m-n}}{(N / \pi) \sin [\pi(m-n) / N]}=t_{n, m}^{l *}
$$

with $t$, a real constant. Also in Eq. (6) down spin hopping integral is taken as negative with respect to that of the up spin hopping. This sign reversal for opposite spins along with the imaginary hopping is responsible for the helical behavior of the ring. It can also be interpreted as the time-reversal counter part as well. On the other hand, the contribution from the Rashba spin-orbit coupling is given by the hopping term ${ }^{10,62,63}$,

$$
\mathbf{t}_{m, n}^{\prime}=-i t_{r s o}\left(\cos \phi_{m, n} \boldsymbol{\sigma}_{x}+\sin \phi_{m, n} \boldsymbol{\sigma}_{y}\right)
$$

with

$$
\phi_{m, n}=\left(\phi_{m}+\phi_{n}\right) / 2,
$$

$\phi_{m}=[2 \pi(m-1) / N]$ being the azimuthal angle for $m$-th site. $t_{r s o}$ is the strength of the hopping integral due to Rashba spin-orbit coupling. $\boldsymbol{\sigma}_{x(y)}$ is the Pauli spin matrix. The effect of magnetic flux is incorporated through the Peierl's phase factor $\Theta_{m, n}$ which can be expressed for the long-range hopping as ${ }^{64}$,

$$
\Theta_{m, n}=\frac{2 \pi|m-n| \Phi}{N \Phi_{0}}
$$

$\Phi_{0}$ being the flux quantum.

Similar to the ring we write the Hamiltonian for the two 1D leads in Wannier basis as,

$$
\begin{aligned}
\boldsymbol{H}_{\mathrm{L}_{1(2)}}= & \epsilon_{0} \sum_{m_{1(2)}} \mathbf{b}_{m_{1(2)}}^{\dagger} \mathbf{b}_{m_{1(2)}} \\
& +t_{0} \sum_{m_{1(2)}}\left(\mathbf{b}_{m_{1(2)}}^{\dagger} \mathbf{b}_{m_{1(2)}+1}+\text { h.c. }\right)
\end{aligned}
$$

where $\epsilon_{0}$ and $t_{0}$ are the on-site energy and nearestneighbor hopping integral for the leads. We have used another notation for the creation $\left(\mathbf{b}_{m_{1(2)}}^{\dagger}\right)$ and annihilation operators $\left(\mathbf{b}_{m_{1(2)}}\right)$ for the leads. They are expressed in similar way as that of $\mathbf{c}_{m_{1(2)}}^{\dagger}$ and $\mathbf{c}_{m_{1(2)}}$ (see Eq. (3)). The ring-to-lead couplings for the two leads are described as,

$$
\boldsymbol{H}_{\mathrm{L}_{1(2)} \mathrm{R}}=\tau\left(\boldsymbol{c}_{m}^{\dagger} \boldsymbol{b}_{m_{1(2)}}+\text { h.c. }\right) \text {. }
$$

$m$ and $m_{1(2)}$ are indices corresponding to the neighboring sites situated at the boundaries of the ring and lead-1(2). $\tau$ is the coupling strength between the lead and the ring.

\section{CALCULATION OF TRANSMISSION PROBABILITY: GREEN'S FUNCTION FORMALISM}

In order to calculate the transmission probability of the incoming electron through the helical ring we adopt Green's function formalism ${ }^{61}$ which is summarized below.

First, let us define the single-particle retarded (advanced) Green's function for our model (helical ring with two side-attached leads) as,

$$
\boldsymbol{G}^{r(a)}=\left(z^{ \pm} \boldsymbol{I}-\boldsymbol{H}\right)^{-1}
$$

where $z^{ \pm}=(E \pm i \eta)$ and $\eta \rightarrow 0^{+} . E$ is the incoming electron energy. Similar to the Hamiltonian, we can also partition the Green's function for the entire system corresponding to the different parts of it like,

$$
\boldsymbol{G}^{r}=\left(\begin{array}{ccc}
\boldsymbol{G}_{\mathrm{L}_{1}} & \boldsymbol{G}_{\mathrm{L}_{1} \mathrm{R}} & 0 \\
\boldsymbol{G}_{\mathrm{L}_{1} \mathrm{R}}^{\dagger} & \boldsymbol{G}_{\mathrm{R}} & \boldsymbol{G}_{\mathrm{RL}_{2}} \\
0 & \boldsymbol{G}_{\mathrm{RL}_{2}}^{\dagger} & \boldsymbol{G}_{\mathrm{L}_{2}}
\end{array}\right) .
$$

Now, we map $\boldsymbol{G}^{r}$ corresponding to the full Hilbert space of the entire system to the reduced Hilbert space which consists of the ring alone. This allows us avoiding the calculation of the infinite-dimensional Green's function for the full system comprised of finite dimensional ring and two semi-infinite leads. After calculation we have the effective Green's function for the isolated ring as follows,

$$
\mathcal{G}^{r}=\left(z^{+} \boldsymbol{I}-\boldsymbol{H}_{R}-\boldsymbol{\Sigma}_{\mathrm{L}_{1}}^{r}-\boldsymbol{\Sigma}_{\mathrm{L}_{2}}^{r}\right)^{-1} .
$$

where

$$
\boldsymbol{\Sigma}_{\mathrm{L}_{1(2)}}^{r}=\boldsymbol{H}_{\mathrm{L}_{1(2)}}^{\dagger} \boldsymbol{g}_{\mathrm{L}_{1(2)}}^{r} \boldsymbol{H}_{\mathrm{L}_{1(2)} R} .
$$

$\boldsymbol{g}_{\mathrm{L}_{1(2)}}^{r}$ is the Green's function for the lead-1(2) defined as,

$$
\boldsymbol{g}_{\mathrm{L}_{1(2)}}^{r}=\left(z^{+} \boldsymbol{I}-\boldsymbol{H}_{\mathrm{L}_{1(2)}}\right)^{-1} .
$$

Thus, dimension of the effective Green's function is same as that of the ring alone. Now, it is sufficient to consider only the isolated ring with two modified boundary sites characterized by effective site-potentials. After 
the mapping, we have two terms $\boldsymbol{\Sigma}_{\mathrm{L}_{1}}^{r}$ and $\boldsymbol{\Sigma}_{\mathrm{L}_{2}}^{r}$. They are the retarded self-energies responsible for the lead-1to-ring and ring-to-lead-2 couplings, respectively. They have non-zero elements only for the boundary sites where the lead(s) and the ring are connected to each other. The self-energy for each lead is expressed in terms of the bare Green's function of the corresponding lead. We calculate them considering both the leads as single channel semiinfinite periodic chains having only nearest neighbor hopping. Expressions for those self-energies in terms of the incoming electron energy and the hopping strengths are given by ${ }^{61}$,

$$
\begin{aligned}
& \boldsymbol{\Sigma}_{L_{\alpha} m \sigma, m^{\prime} \sigma^{\prime}}^{r}(E) \\
= & \frac{\tau^{2}}{2 t_{0}^{2}} \delta_{m m_{\alpha}} \delta_{m^{\prime} m_{\alpha}} \delta_{\sigma \sigma^{\prime}}\left[z^{+}-\epsilon_{0}-i \sqrt{4 t_{0}^{2}-\left(z^{+}-\epsilon_{0}\right)^{2}}\right] .
\end{aligned}
$$

Here, $\alpha$ is the lead index. $m$ and $m_{\alpha}$ are the site indices in the ring and $\alpha$-th lead, respectively. Note that, the self-energies corresponding to the two leads have both finite real and imaginary parts contributing to the effective Green's function. The real part is responsible for the energy level shifting whereas, the imaginary part describes the broadening of the levels. In other words, one can separate out the broadening matrix parts corresponding to each $\sigma$ like,

$$
\boldsymbol{\Gamma}_{\mathrm{L}_{1(2)}}^{\sigma}=-2 \operatorname{Im}\left(\boldsymbol{\Sigma}_{\mathrm{L}_{1(2)} \sigma}^{r}\right)
$$

Now, in terms of the broadening matrices and the effective Green's functions, the transmission function can be found from the following relation ${ }^{61}$,

$$
T_{\sigma \sigma^{\prime}}(E)=\operatorname{Tr}\left[\boldsymbol{\Gamma}_{\mathrm{L}_{1}}^{\sigma} \cdot \mathcal{G}^{r} \cdot \boldsymbol{\Gamma}_{\mathrm{L}_{2}}^{\sigma^{\prime}} \cdot \mathcal{G}^{a}\right]
$$

$T_{\sigma \sigma^{\prime}}$ represents the transmission probability of the incoming electron through lead-1 with spin $\sigma$ as an electron with spin $\sigma^{\prime}$ into lead-2. For the advanced part of the effective Green's function, one can take the complex conjugate of the expression written in Eq. (15).

\section{NUMERICAL RESULTS}

For numerical calculation, we use the unit where $c=$ $h=e=1$. The hopping parameter $t$ is taken as the unit of energy. Throughout our calculation we take the ring parameter values as, $N=91, t=2, a=1$ and for clean system $\epsilon=0$; the lead parameters as, $\epsilon_{0}=0, t_{0}=3$. The leads are attached to the sites $n_{\mathrm{L}_{1}}$ and $n_{\mathrm{L}_{2}}$, respectively with the coupling strength $\tau=2.5$ for both leads signifying strong lead-to-ring coupling. We set them as, $n_{\mathrm{L}_{1}}=1$ and $n_{\mathrm{L}_{2}}=46$. Now, we illustrate our results in three different sub-sections in order to describe the effect of magnetic flux, Rashba SOI and scalar disorder successively.

\section{A. Effect of magnetic flux}

In Fig. 2 we plot the energy levels of the ring as a function of $\mathrm{AB}$ flux $\Phi$. We take an isolated ring having 91 number of lattice sites and diagonalize the Hamiltonian to find the energy levels. Fig. 2(a) represent the entire spectrum of the isolated ring. We zoom-in the spectrum around $\Phi=0$ and $\Phi=\Phi_{0} / 2$ and present them in Fig. 2(b) and Fig. 2(c), respectively.

The most remarkable feature is that there is zero energy crossing at $\Phi=0$. Similar zero energy crossing in the energy spectrum of this helical ring model was also articulated in Ref. 43 in absence of magnetic flux.
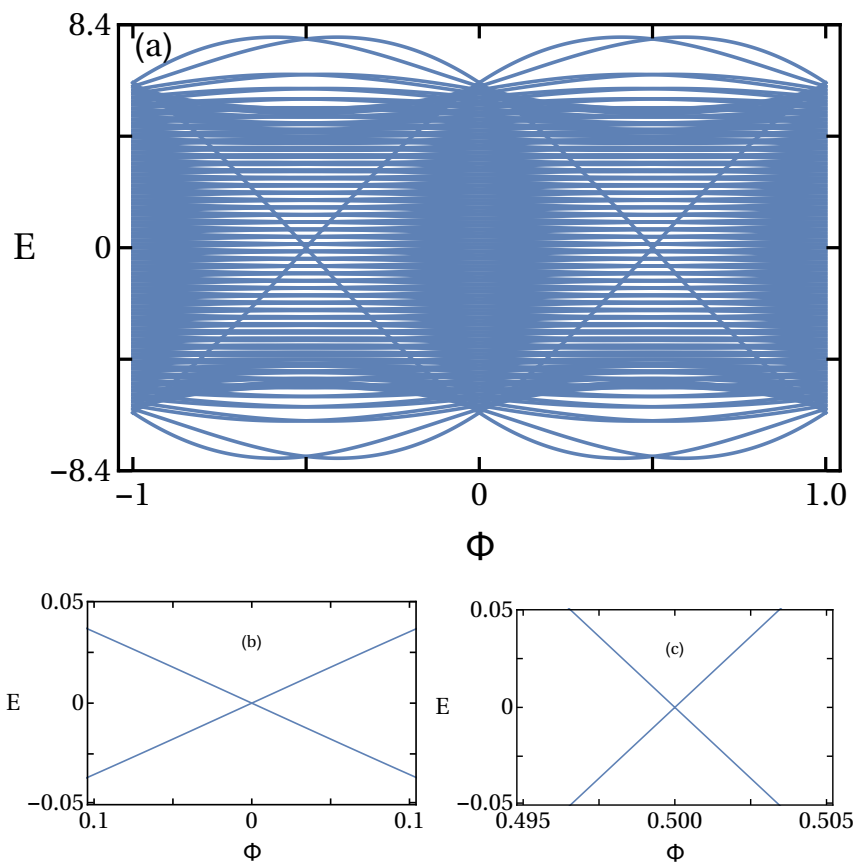

FIG. 2. (Color online) Upper panel: (a) Full energy spectrum $(E-\Phi)$ of a clean ring with atomic sites $N=91$. Lower Panel: The zoomed-in versions of the full spectrum around (b) $\Phi=0$ and (c) $\Phi=\Phi_{0} / 2$.

This zero energy crossing associated with two opposite spin states is analogous to the linear dispersion relation of the topological insulator edge states ${ }^{32,33,40}$. An analytic derivation of the dispersion relation ( $E$ vs. $k$ ) of the isolated ring in presence of $\mathrm{AB}$ flux is presented in Appendix.

In addition to $\Phi=0$ we get similar zero energy crossings at all integer multiples of half-flux quantum values of the applied AB flux. Due to the crossing we have both the positive and negative slopes of the curves i.e. both positive and negative group velocities are possible for the electrons with two opposite spins and this results in appearance of counter-propagating currents characterized by two opposite spin states. To ensure the current direction corresponding to two spins one can calculate the bond current between two sites along any arm of the ring 

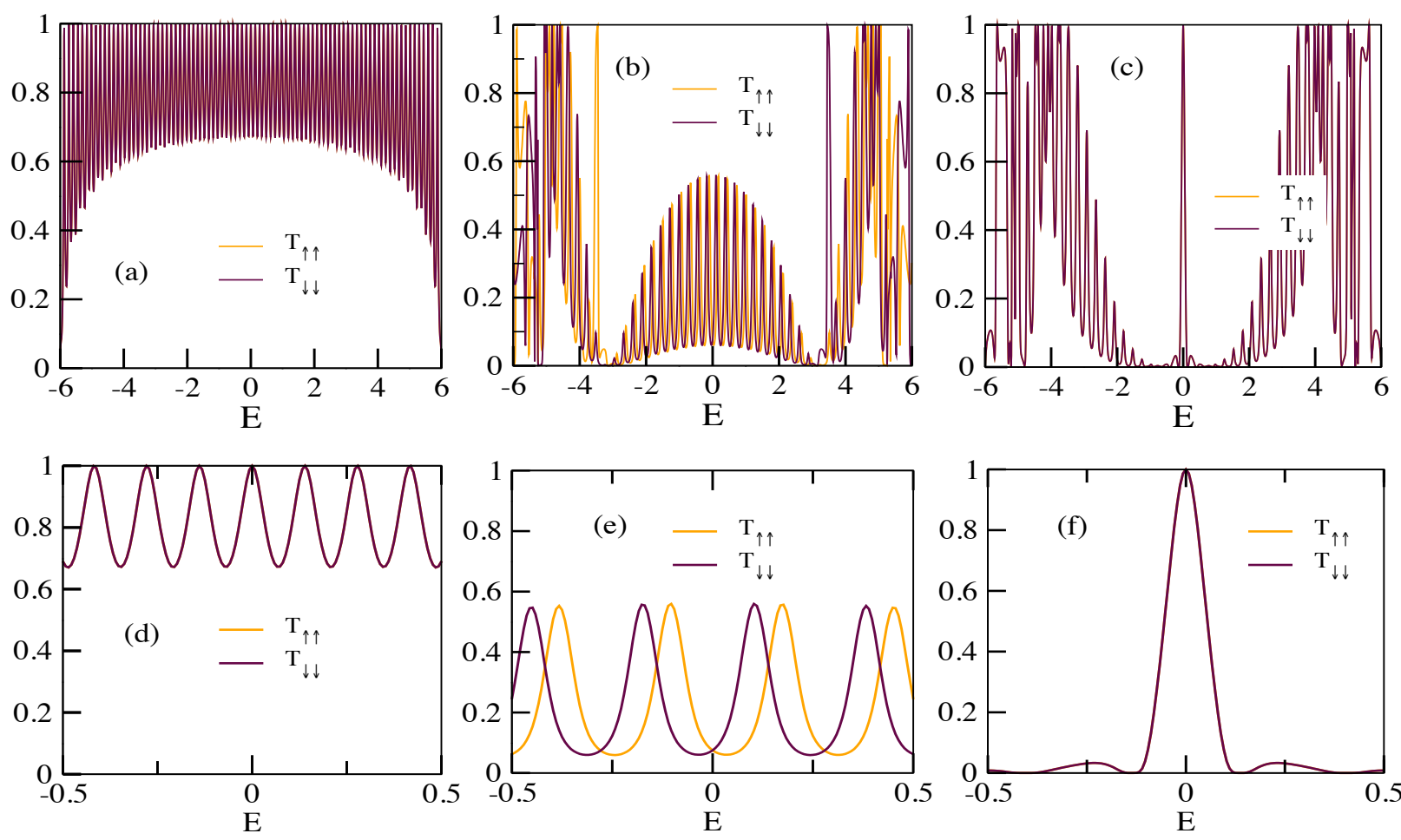

FIG. 3. (Color online) Quantum-mechanical transmission probabilities $T_{\uparrow \uparrow}$ (orange color) and $T_{\downarrow \downarrow}$ (maroon color) are plotted as a function of incoming electron energy $E$ corresponding to a ring with $N=91$ for three values of $\mathrm{AB}$ flux (a) $\Phi=0$, (b) $\Phi=\Phi_{0} / 4$ and (c) $\Phi=\Phi_{0} / 2$. (d), (e) and (f) represent the zoomed-in versions of (a), (b) and (c) respectively around $E=0$.

considering the up and down spin electrons ${ }^{65}$. We skip this part as one can easily guess the two opposite signs of the currents by looking at the signs of the hopping integral as given in Eq. (6).

To investigate the outcome of the helical states with the applied magnetic flux we study the transmission probability of electrons through the ring. We compute the transmission probability of electrons following Eq. (20) for both up and down spin electrons and show their behaviors in Fig. 3 with respect to the incoming electron energy $E$. The maroon and orange color represent $T_{\uparrow \uparrow}$ and $T_{\downarrow \downarrow}$ i.e. the probability of transmission of the $\uparrow$ and $\downarrow$ spin electrons without any spin-flip scattering. Spin flipping is not possible as we have not included the Rashba spin-orbit interaction so far. Here, Figs. $3[(\mathrm{a}),(\mathrm{b})$ and $(\mathrm{c})]$ correspond to the three different values of the $\mathrm{AB}$ flux $\Phi=0, \Phi_{0} / 4$ and $\Phi_{0} / 2$, respectively. We zoom into all the three figures ((a)-(c)) around $E=0$ and display them in Figs. 3 [(d), (e) and (f)], respectively.

In absence of any flux $(\Phi=0)$ we note that transmission curve shows oscillatory behavior (see Fig. 3(a)). The oscillation is much more prominent in the zoomedin version (see Fig. 3(d)). Our model ring is attached to two leads. So, the wave functions corresponding to the incoming electrons pass through the two arms of the ring. After traveling through the two arms they again meet at the junction, where the lead- 2 is attached to the ring, either constructively or destructively. This quan- tum interference leads to an oscillatory behavior of the transmission probability. In the oscillation, number of peaks describing the resonances is equal to the number of energy levels of the isolated ring. To be mentioned, we consider completely unpolarized beam for the incoming electrons.

However, this phenomenon of quantum interference is true for both the up and down spin electrons. Only, the difference between them is that they travel along two opposite directions within the ring. The transmission probabilities corresponding to two spin states of the electrons are exactly superposed on each other as the time-reversal symmetry between the $\uparrow$ and $\downarrow$ spin states is protected. Our result for $\Phi=0$ (see Fig. 3(d)) corroborates with the results obtained in Ref. 43 where the energy dependence of transmission probability of electrons is reported. Although the frequency of the oscillation being different as it depends on the ring size. However, the most interesting feature of the transmission spectra is that there are zero-energy peaks for both the spin states and these peaks correspond to the zero energy crossing in the energy spectra at $\Phi=0$ as shown in Fig. 2(b).

Now, as soon as we introduce $\mathrm{AB}$ flux $\Phi$, the time reversal symmetry between the up and down spin states breaks down i.e. the symmetry between left-moving up spin and right-moving down spin electrons no longer exists. A phase difference is introduced between the wave functions corresponding to the clockwise and anti- 
clockwise propagating electrons with opposite spins. As a result, in the feature of transmission probabilities the oscillations corresponding to $T_{\uparrow \uparrow}$ and $T_{\downarrow \downarrow}$ are different from each other by a phase factor (see Fig. 3(b)). However, the amplitudes of oscillation are not same throughout the energy window because of the destructive interference between the wave functions propagating along the two arclengths. The removal of the degeneracy between $\uparrow$ and $\downarrow$ is much more prominent in Fig. 3(e). This leads to a possibility of obtaining finite spin polarization depending on the energy value and applied $\mathrm{AB}$ flux. It should be noted, there is no zero-energy peak in the transmission spectra for this flux value. This can be understood by looking at the energy spectra around this flux value as depicted in Fig. 2(a).

Nonetheless, this phenomenon of separation of the transmission curves corresponding to the two different spin states by a phase difference is true as long as the flux value is different from the half-flux quantum. To investigate this, in Fig. 3(c) we plot the probability of $\uparrow$ and $\downarrow$ spin electron transmission vs. energy $E$ for $\Phi=\Phi_{0} / 2$ choosing all the other parameter values same as in the previous two cases for other two flux $(\Phi)$ values. We observe that when $\Phi=\Phi_{0} / 2, T_{\uparrow \uparrow}$ and $T_{\downarrow \downarrow}$ are again exactly superposed on each other nullifying the possibility of getting finite spin polarization. Moreover, we have a peak at exactly $E=0$ and this peak corresponds to the crossing at zero energy for $\Phi=\Phi_{0} / 2$ as shown in Fig. 2(c). The persistence of the zero energy peak correspond to the spinor nature of the electrons. However, the appearance of the zero-energy peaks at particularly these flux values (i.e. $\pm \Phi_{0} / 2$ ) is completely a topological signature of the helical ring with the underlying inversion symmetry. They neither depend on the details (different sizes of the two ring arms) of the ring nor the position of the attached leads. Note that, there is no Zeeman effect in our case. We only consider a single channel of the ring. Zeeman splitting does not play any role as far as the radius of the ring is less than the radius of the cyclotron orbit. In our case, only the $\mathrm{AB}$ effect is the effective one.

To analyze the spin polarization in detail we define a function, namely the spin polarization factor $\mathrm{as}^{24}$,

$$
P_{\uparrow(\downarrow)}=\frac{T_{\uparrow \uparrow(\downarrow \downarrow)}-T_{\downarrow \downarrow(\uparrow \uparrow)}}{T_{\uparrow \uparrow}+T_{\downarrow \downarrow}}
$$

where $P_{\uparrow}$ and $P_{\downarrow}$ correspond to the two different spin states $\uparrow$ and $\downarrow$. Their behaviors with respect to the incoming electron energy are shown in Fig. 4 where panel (a) and (b) correspond to $\Phi=\Phi_{0} / 4$ and $\Phi_{0} / 2$ respectively. The blue and red lines represent the efficiency factors for $\uparrow$ and $\downarrow$ spin electrons, respectively. From Fig. 4(a) we see that both $P_{\uparrow}$ and $P_{\downarrow}$ have oscillatory behaviors being dual to each other. Their amplitudes run between 0.7 and -0.7 approximately whereas the maximum allowed values for the amplitudes are \pm 1 . Looking at the expressions for the efficiency factors we can say that $P_{\uparrow}=1$ indicates that $T_{\downarrow \downarrow}$ is exactly equal to zero but $T_{\uparrow \uparrow}=1$. That means we have only $\uparrow$ spin electron
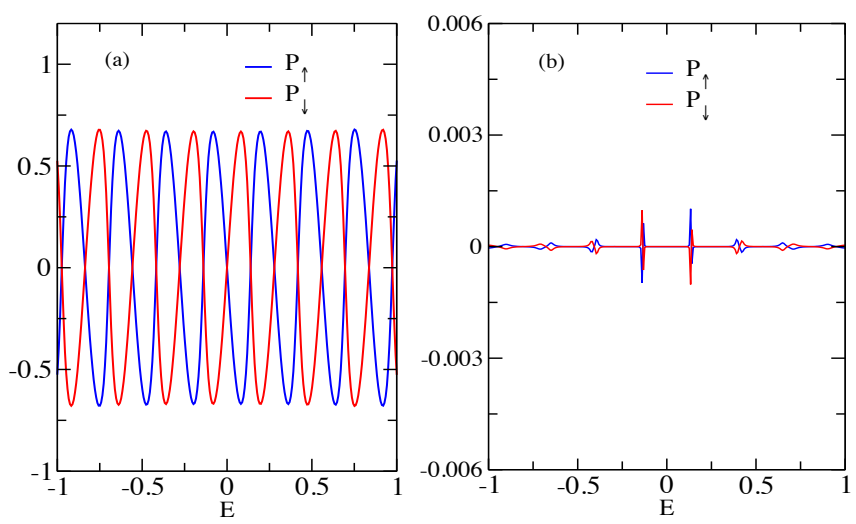

FIG. 4. (Color online) Spin polarization is shown as a function incident electron energy $E$ for up $\left(P_{\uparrow}\right)$ and down $\left(P_{\downarrow}\right)$ spin electrons with $\operatorname{AB}$ flux (a) $\Phi=\Phi_{0} / 4$ and (b) $\Phi_{0} / 2$ respectively.

transmission. The transmissions of $\downarrow$ spin electrons are completely blocked for that particular energy value. In contrast, when $P_{\downarrow}=1$ we have exactly the opposite scenario. However, in our case amplitude of the $P_{\sigma}$ curve is almost 0.7 i.e. all the energy values where $P_{\downarrow}=0.7$ we have $70 \%$ polarization with $\downarrow$ spin being favorable one, suppressing $\uparrow$ spin transmission and vice-versa. For the rest of the energy values the spin polarization is less than $70 \%$.

From the oscillations of $P_{\sigma}$ ( $\sigma$ may be $\uparrow$ or $\downarrow$ ) we obtain some energy values where transition happens i.e. the efficiency factors change their signs. Now, the change in sign of one efficiency factor, say $P_{\sigma}$, signifies that the transmission of electrons having opposite spin starts dominating. Note that, we have shown the result for a particular energy range. Although for the entire energy band we get similar features of $P_{\uparrow}$ and $P_{\downarrow}$. Therefore, for a particular flux value the spin polarization is different for different energy values or in other words, spin polarization of the helical ring is energy-dependent.

On the other hand, if we tune the flux we get different behaviors of the efficiency factors for a particular energy value. To examine this, we calculate the efficiency factors for $\Phi=\Phi_{0} / 2$. The up and down spin electron transmission again become exactly symmetric to each other resulting in vanishing spin polarization at this flux value. This is clear from Fig. 4(b) where both $P_{\uparrow}$ and $P_{\downarrow}$ are vanishingly small $\left(\sim 10^{-4}\right)$ in magnitude. However, the deviation of the efficiency value $\left(P_{\uparrow}\right.$ or $\left.P_{\downarrow}\right)$ from exact zero occurs due to the finite size of the ring.

The most striking feature is that we obtain finite spin polarization using the long-range hopping model just by tuning the magnetic flux. If we fix the magnetic flux to a finite value other than half-flux quantum we get finite spin polarization by tuning the applied bias. In other words, we can tune the magnetic flux to have the spin polarization finite or zero for a particular energy value. This is in contrast to the behavior of ordinary periodic ring. In ordinary ring with nearest-neighbor hopping, when we 
apply magnetic flux the transmission is modified as a result of the quantum interference of the electronic wave functions traveling along the two arms of the ring. However, one cannot break the spin degeneracy just by tuning the applied AB flux. So, the separation between the up and down spin transmission is not possible ${ }^{24}$. They are exactly symmetric to each other.

\section{B. Effect of Rashba spin-orbit interaction}

In this sub-section we investigate the effect of RSOI on the transport properties of the helical ring with longrange hopping. Fig. 5 displays the energy spectrum $(E-$ $\Phi)$ of the isolated ring with $N=91$ in presence of RSOI with strength $t_{r s o}=1.5$. We observe that there is a gap at the central region (i.e. around $\Phi=0$ ) of the spectrum. In absence of RSOI we have a zero-energy crossing at $\Phi=0$ and the crossing no longer exists in presence of Rashba spin-orbit interaction. This gap arises due to spin flip scattering which makes this helical ring to behave like an ordinary insulator at $\Phi=0$. Such gap may seem to
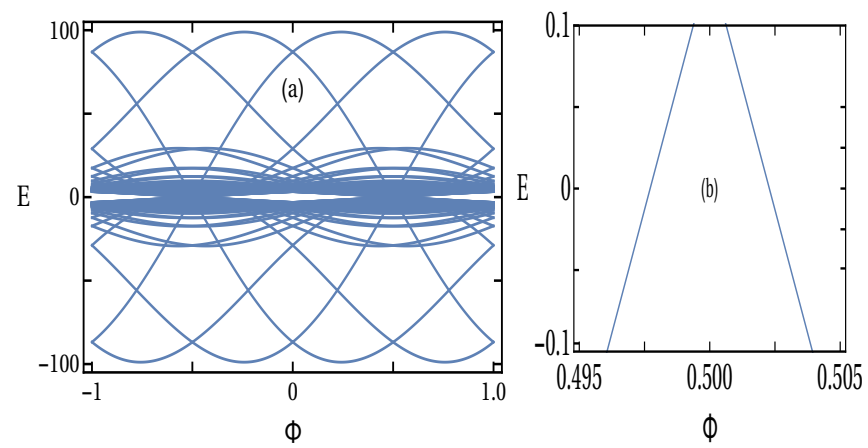

FIG. 5. (Color online) Left column (a): Full energy spectrum $(E-\Phi)$ of a clean ring $(N=91)$ in presence of RSOI $\left(t_{r s o}=\right.$ 1.5). Right column (b): Part of the spectrum of Fig. 5(a) while zoomed-in around $\Phi=\Phi_{0} / 2$.

appear due to the destructive interference caused by the incomplete Rashba spin flipping along the chain. Also, constructive intereference is possible for very small size of the ring and this can lead to the closing of the gap around $\Phi=0$. To overcome this effect we have taken more than 75 number of sites in the ring. We obtain wider energy band and this enhancement can be realized more prominently by comparing with Fig. 2. The splitting for outer bands is higher compared to that of the inner bands. We also zoom-in the spectrum around $\Phi=\Phi_{0} / 2$. We have energy levels at $E=0$ corresponding to which we expect finite transmission but there is no crossing at this flux value similar to the case of $\Phi=0$. In topological insulator or the spin quantum Hall states we find helical edge states that disperse linearly. In these systems Rashba spin-orbit interaction is an inherent property. In our 1D ring with long-range hopping we see that such Dirac-like crossings do not exist in spectrum in presence of RSOI. When we incorporate Rashba spin-orbit interaction all the energy levels get split. This results in a gap in the spectrum. For finite values of $\Phi$, we have two contributions to the phase part, one due to the magnetic flux and another due to Rashba spin-orbit interaction. There may be compensation of the phase due to one by the other resulting in a shifting of energy levels. Therefore, we loss the zero energy crossing at the half flux quantum values also.

To be noted, a minute asymmetry $(\sim 0.05 \%)$ appears around $E=0$ in Fig. 5(b). This asymmetry is fully due to the finite-size effect of the ring which causes an overall shifting of the energy spectra from the zero energy value. It does not depend on the flux value. The particle-hole symmetry is preserved in our system even in presence of RSOI.

Now, we study the transmission phenomena in presence of RSOI. In Fig. 6 we show all four possible spin
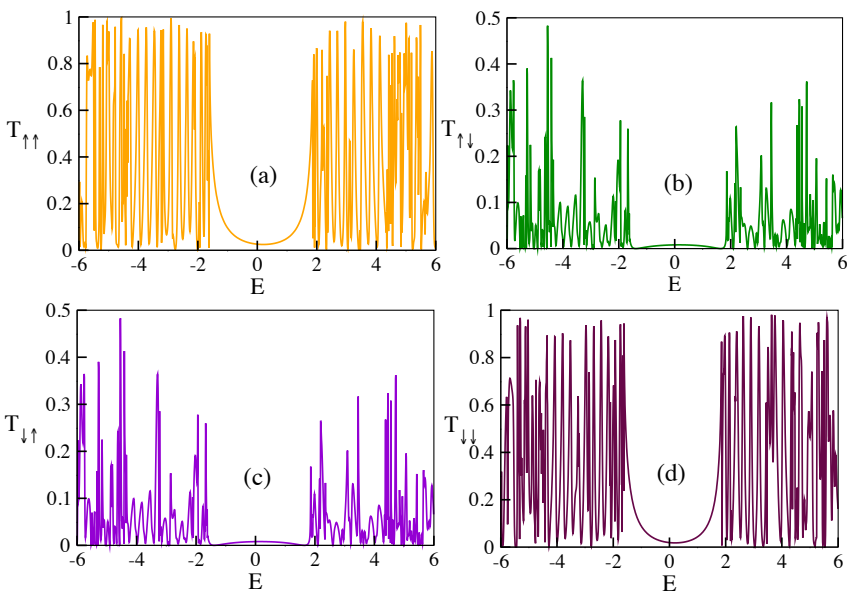

FIG. 6. (Color online) Plot of transmission probabilities ((a) $T_{\uparrow \uparrow}$, (b) $T_{\uparrow \downarrow}$, (c) $T_{\downarrow \uparrow}$, and (d) $T_{\downarrow \downarrow}$ ) as a function of incoming electron energy $E$ for a ring with $N=91$ in presence of RSOI $\left(t_{r s o}=1.5\right)$ and $\Phi=\Phi_{0} / 4$.

transmission probabilities as a function of injecting electron energy $E$ in presence of magnetic flux $\Phi=\Phi_{0} / 4$. Fig. $6[(\mathrm{a}),(\mathrm{b}),(\mathrm{c})$ and $(\mathrm{d})]$ correspond to $T_{\uparrow \uparrow}$ (orange lines), $T_{\uparrow \downarrow}$ (green lines), $T_{\downarrow \uparrow}$ (violet lines) and $T_{\downarrow \downarrow}$ (maroon lines) respectively. Now, we see that the probability of $\uparrow$ and $\downarrow$ spin electron transmissions without any spin flipping are comparable in magnitude but they are not exactly equal to each other. There are central gaps around $E=0$ in the both spectra of $T_{\uparrow \uparrow}$ and $T_{\downarrow \downarrow}$. With the increase of Rashba hopping strength these gap-widths increase. On the other hand, due to presence of RSOI we have finite spin-flip transmission probability for both up and down spin electrons as shown in Fig. 6(b) and Fig. 6(c) respectively. Note that, the magnitude of spinflip transmission probability is much smaller compared to the spin-conserving transmission probabilities. Also, there are very small probability of transmission of elec- 
trons at $E=0$ for this flux value.

RSOI actually behaves like an effective magnetic field which depends on the momenta of the electrons. It causes a phase difference between the electronic wave functions corresponding to the two spin states traveling along opposite directions. This induced phase difference modifies the quantum interference phenomenon both constructively as well as destructively. As a consequence, we get different magnitudes of the transmission probabilities for any particular energy value. It is evident while we compare Figs. 3[(a)-(c)] and Figs. 6. Whereas, for $\Phi=\Phi_{0} / 2$,
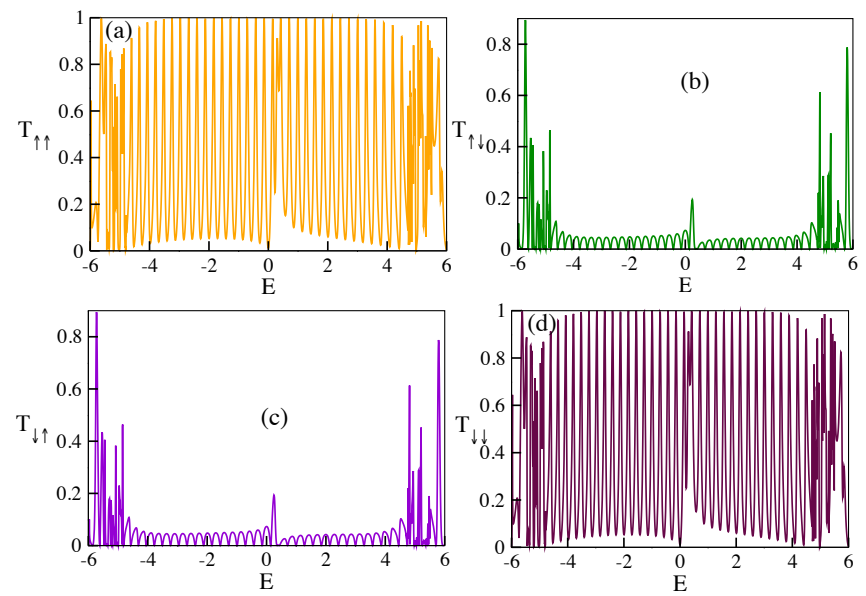

FIG. 7. (Color online) Quantum-mechanical transmission probabilities (a) $T_{\uparrow \uparrow}$, (b) $T_{\uparrow \uparrow}$, (c) $T_{\uparrow \uparrow}$, and (d) $T_{\downarrow \downarrow}$ are shown as a function of incoming electron energy $E$ for $\Phi=\Phi_{0} / 2$. The values of other parameters of the ring are kept same as in Fig. 6.

we have already seen from the energy spectra that there are energy levels available at $E=0$. This reflects in the transmission spectra accordingly. We have finite spin-flip transmission at this energy value. In Fig. 7 we display the four transmission probabilities of electrons through the ring keeping all the parameter values same as in Fig. 6 . It looks that the central gaps of the spectra of $T_{\uparrow \uparrow}$ and $T_{\downarrow \downarrow}$ disappear for $\Phi=\Phi_{0} / 2$. If we observe carefully then we notice that there are dips at $E=0$ in the spectra of electron transmission without spin-flipping. If we change the RSOI strength there may be finite spin transmission at this energy value in place of a central gap. We can say that the zero energy peak no longer exist when $\Phi=\Phi_{0} / 2$ also. Additionally, $T_{\uparrow \uparrow}$ and $T_{\downarrow \downarrow}$ become exactly symmetric to each other. On the other hand, spinflip electron transmission is finite throughout the energy window. Therefore, in presence of RSOI zero-peaks associated with the zero energy crossings are absent for any value of magnetic flux.

From all the figures of the transmission probabilities in presence of RSOI we can argue that the possibility of getting finite spin polarization is extremely small. We do not plot the spin polarization here. On the contrary, in case of ordinary ring one can design a spin-filter device based on the spin polarization just by tuning the RSOI alone $^{24}$. Note that, the small asymmetry around $E=0$ in each plot of the transmission spectra appears because of the asymmetry in the energy spectra originating due to the finite size of the discrete model.

\section{Effect of disorder}

The helical states obtained at the edges of a $2 \mathrm{D}$ topological insulator are robust to non-magnetic impurity $^{28,29,31}$. Here we investigate whether the electron flows corresponding to the opposite spins traveling in opposite directions along each arm of the ring get affected by static disorder or not. For this, we introduce static scalar random disorder into the system. This type of disorder does not affect the spin state of electrons. We choose the on-site potential energies of the ring ran-
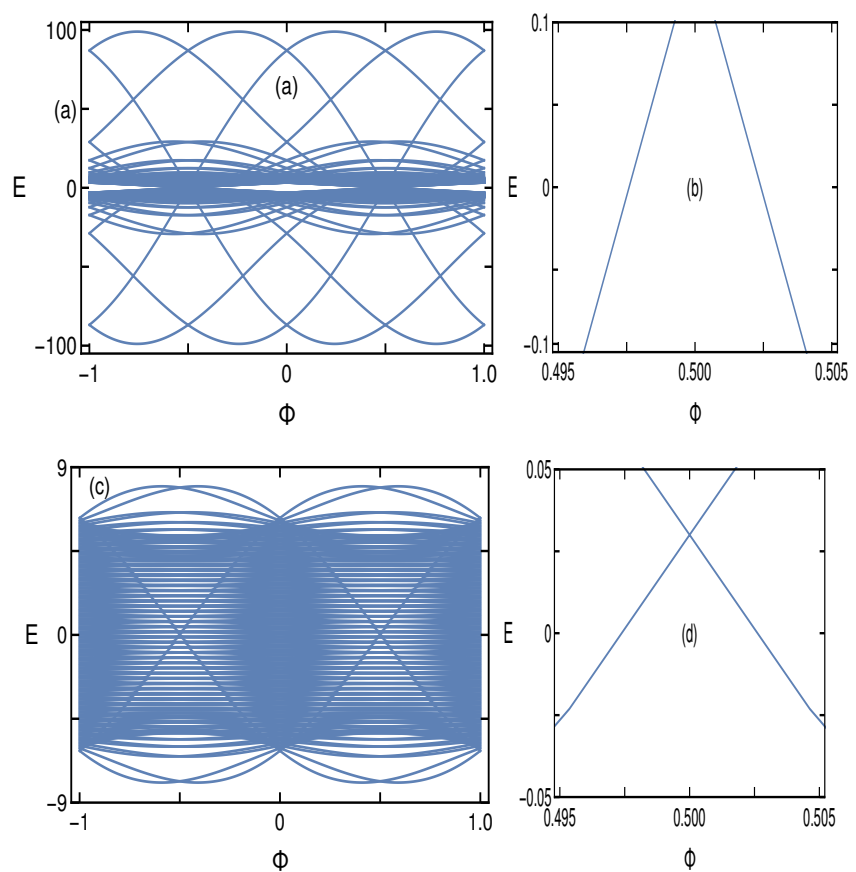

FIG. 8. (Color online) Left panel: Plot of energy spectra ( $E$ $\Phi)$ for the disordered ring $(N=91)$ with disorder strength $W=3$ in presence of RSOI $t_{r s o}=1.5$ (a) and in absence of RSOI $t_{r s o}=0$ (c). Right panel: Illustrations of figure (a) and (c) around $E=0$ are presented in figure (b) and (d) respectively.

domly from a "Box" distribution function within the range $(-W / 4, W / 4)$ describing the disorder strength $W$. In Fig. 8, we show the full energy spectrum i.e. $E$ vs. $\Phi$ both in presence (Fig. 8(a)) and absence (Fig. 8(c)) of RSOI. Their zoomed-in versions around $\Phi=\Phi_{0} / 2$ are presented in Figs. 8(b) and (d) respectively. The full spectrum of the isolated disordered ring looks very much similar to that obtained in absence of disorder, but both in presence and absence of RSOI as compared with Fig. 5(a) and Fig. 2(a), respectively. The disor- 
der strength is taken as $W=3$. Similar to the case of clean system we have also a central gap around $\Phi=0$ in presence of RSOI. There is no such zero-energy crossing at $\Phi=0$ in presence of static disorder also. To examine the spectra at half-flux quantum values we zoom-in the full spectrum around $\Phi=\Phi_{0} / 2$ (see Fig. 8(b)) and
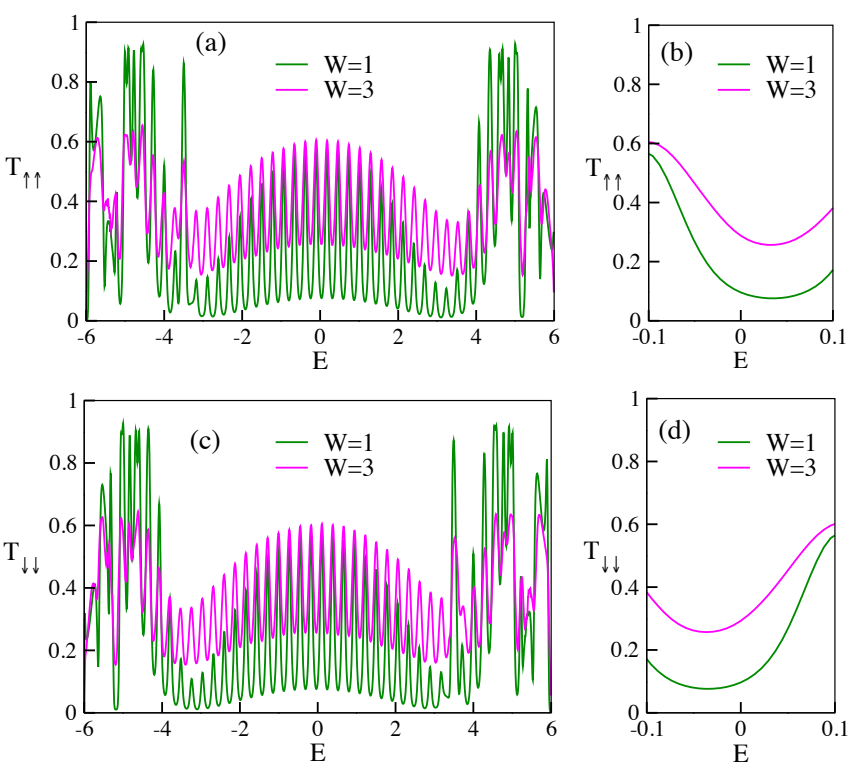

FIG. 9. (Color online) Behaviors of average transmission probabilities (a) $T_{\uparrow \uparrow}$ and (c) $T_{\downarrow \downarrow}$ are displayed as a function of incoming electron energy $E$ for a disordered ring $(N=91)$ with $\Phi=\Phi_{0} / 4$ in absence of $\operatorname{RSOI}\left(t_{r s o}=0\right)$. (b) and (d) represent the zoomed-in diagrams of (a) and (c), respectively. The green and magenta colors correspond to the disorder strength $W=1$ and $W=3$, respectively. We consider that 60 sites of the ring are disordered and the result is averaged over 100 disorder configurations.

observe the absence of zero energy crossings too. Comparing Fig. 8(a) with Fig. 5(a), we observe that the difference between the two figures are very small. The difference will be much more prominent with higher disorder strength. On the other hand, in absence of RSOI, the zero energy crossing gets shifted due to the disorder and the amount of shift depends on the disorder strength (see Fig. 8(d)). That means the zero-energy crossings that we obtain in our helical ring spectra are not immune to disorder. To be noted, we consider 60 out of 91 sites of the ring as disordered.

In order to examine the transmission characteristics of the electrons in presence of static random disorder we plot disorder averaged transmission probabilities, $T_{\uparrow \uparrow}$ and $T_{\downarrow \downarrow}$, in Fig. 9(a) and Fig. 9(c) and their zoomed-in (around $E=0$ ) versions in Fig. 9(b) and Fig. 9(d) respectively. Here, green and magenta colors correspond to $W=1$ and $W=3$, respectively. We consider $t_{r s o}=0$. In this analysis, 2/3-rd of the total number of sites of the ring are taken as disordered sites and the average is taken over 100 random disorder configurations. We notice that with the enhancement of disorder strength, transmission increases upto a certain value of energy and then decreases. Now, in ordinary periodic ring with nearest-neighbor hopping, transmission always decreases as soon as we introduce disorder. The higher the disorder strength the lower is the transmission probability. Now, manifestation of Anderson type localization in 1D disordered systems is very well known ${ }^{66}$. Presence of random site potentials cause localization of the electronic eigenstates which are basically extended in absence of disorder. With the enhancement of the disorder strength the number of localized states increases. As a result transmission probability reduces. The reduction is more pronounced when the disorder strength becomes higher than the hopping integral. Specifically, when $W<<t$, the Bloch waves are weakly scattered by the random potential. On the other hand, for the condition $W>>t$, the electronic
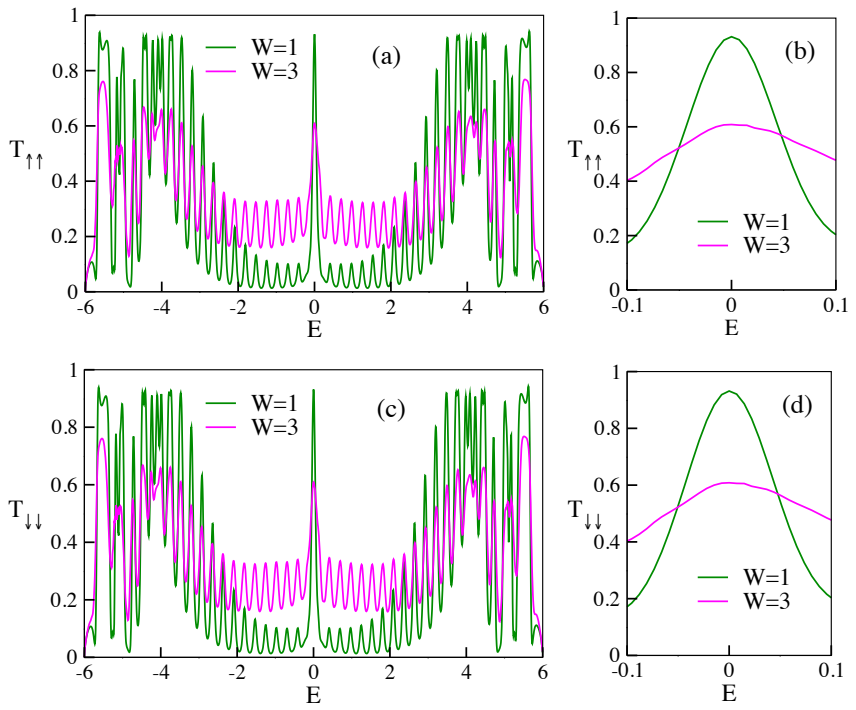

FIG. 10. (Color online) Plots of transmission probabilities (a) $T_{\uparrow \uparrow}$ and (c) $T_{\downarrow \downarrow}$ as a function of incoming electron energy $E$ for a disordered ring are depicted for $\Phi=\Phi_{0} / 2$ in absence of RSOI $\left(t_{r s o}=0\right)$. (b) and (d) represent the zoomed-in diagrams of (a) and (c), respectively. The green and magenta colors indicate the results corresponding to the disorder strength $W=1$ and $W=3$, respectively. Other parameters are fixed to the values same as in Fig. 9 .

states get localized and they fall off as $e^{-r / \xi}, \xi$ being the localization length. The system starts behaving like an insulator ${ }^{67}$.

In contrast to the short-range hopping, when we consider long-range hopping localization phenomenon can not dominate the electrons to transmit extendedly due to the presence of higher order hoppings. Long-range hopping basically induces an infinite number of resonances ${ }^{57}$. As a consequence localization cannot become so effective. Even extended state can be obtained in presence of longrange correlated disordered system ${ }^{47,48}$. From Fig. 9, we observe that with the increase of the disorder strength $W$ the transmission increases upto a certain energy value. Then it decreases with the further increase of disorder 
strength. We cannot predetermine the behavior of the transmission amplitudes. Also, we see that there is no peak at zero-energy value similar to the case of clean system as presented in Fig. 3(e). If we further increase the disorder strength then transmission decreases for all the energy values due to localization of the electronic wave-functions.

We also study the effect of disorder on the electron transmission in helical ring with long-range hopping for other finite values of the $\mathrm{AB}$ flux. For illustration, when we tune the magnetic flux from $\Phi_{0} / 4$ to $\Phi_{0} / 2$ we get similar effect of disorder on the transmission probability. The latter means in presence of long-range hopping whatever may be the value of magnetic flux we cannot predict whether the transmission through the disordered ring would decrease or increase unless we apply very strong disorder. In Figs. 10(a) and (c) we present $T_{\uparrow \uparrow}$ and $T_{\downarrow \downarrow}$, respectively. Whereas Figs. $10(\mathrm{~b})$ and (d) cor-
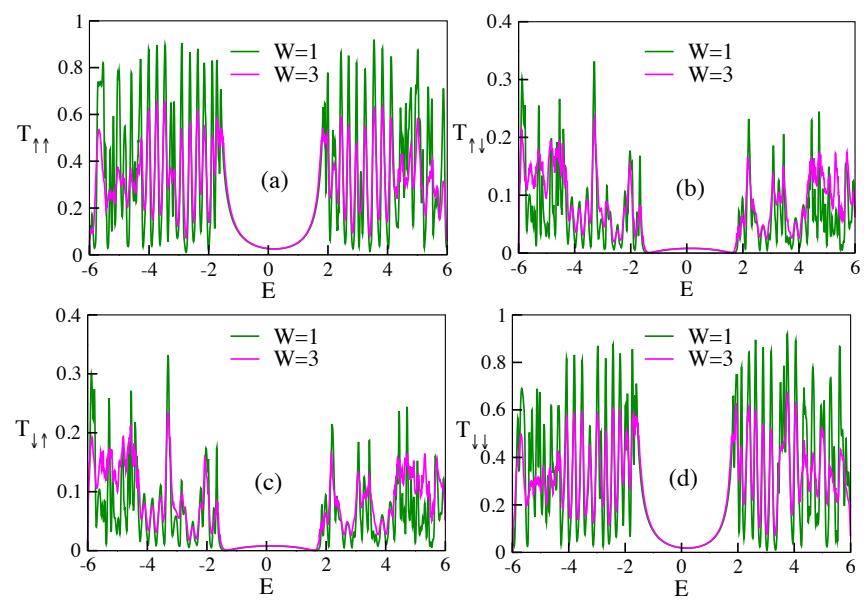

FIG. 11. (Color online) Quantum-mechanical transmission probabilities (a) $T_{\uparrow \uparrow}$, (b) $T_{\uparrow \downarrow}$, (c) $T_{\downarrow \uparrow}$ and (d) $T_{\downarrow \downarrow}$ vs. incoming electron energy $E$ are shown for a disordered ring $(N=91)$ in presence of RSOI $\left(t_{r s o}=1.5\right)$ and $\mathrm{AB}$ flux $\left(\Phi=\Phi_{0} / 4\right)$. The green and magenta colors correspond to disorder strength $W=1$ and $W=3$, respectively. Rest of the parameter values are kept same as in Fig. 9.

respond to their zoomed-in versions around $E=0$. To be noted, the zero-energy peak for $\Phi=\Phi_{0} / 2$ remains as it was when $W<t$. The peak height decreases with the increase of disorder strength. Also, the peak positions get shifted with the rise of the disorder strength. In this figure, we get very slight movement of the peak. If we further increase $W$ the shift will be much more prominent. Moreover, the plots of $T_{\uparrow \uparrow}$ and $T_{\downarrow \downarrow}$ are exactly similar to each other like the phenomenon happened in absence of disorder for $\Phi=\Phi_{0} / 2$.

So far, RSOI has not been taken into consideration in this sub-section while calculating the transmission probabilities in presence of disorder. In Fig. 11 we show the results in presence of RSOI $\left(t_{r s o}=1.5\right)$ and $\mathrm{AB}$ flux $\Phi=\Phi_{0} / 4$. Figs. 11[(a), (b), (c) and (d)] correspond to $T_{\uparrow \uparrow}, T_{\uparrow \downarrow}, T_{\downarrow \uparrow}$ and $T_{\downarrow \downarrow}$, respectively. The green and ma- genta colors have the same meaning as in the previous two figures. In presence of RSOI, we have finite spin-flip transmission probabilities for both $T_{\uparrow \downarrow}$ and $T_{\downarrow \uparrow}$ due to the finite possibility of spin-flipping of electrons. Now, from Fig. 11 we notice that in the disordered helical ring when we increase the disorder strength all the four transmission probabilities decrease in presence of $\mathrm{AB}$ flux. This phenomenon is very much similar to the case of periodic ring with only nearest-neighbor hopping ${ }^{13}$. From literature, we know that RSOI itself may give rise to localization of electronic wave functions. This localization phenomenon can be manifested as the spin-precession due to Rashba field alone ${ }^{68}$. The overall effect of $\mathrm{AB}$ flux and RSOI is the reduction of the transmission of electrons. To be mentioned, in presence of static disorder the cen-
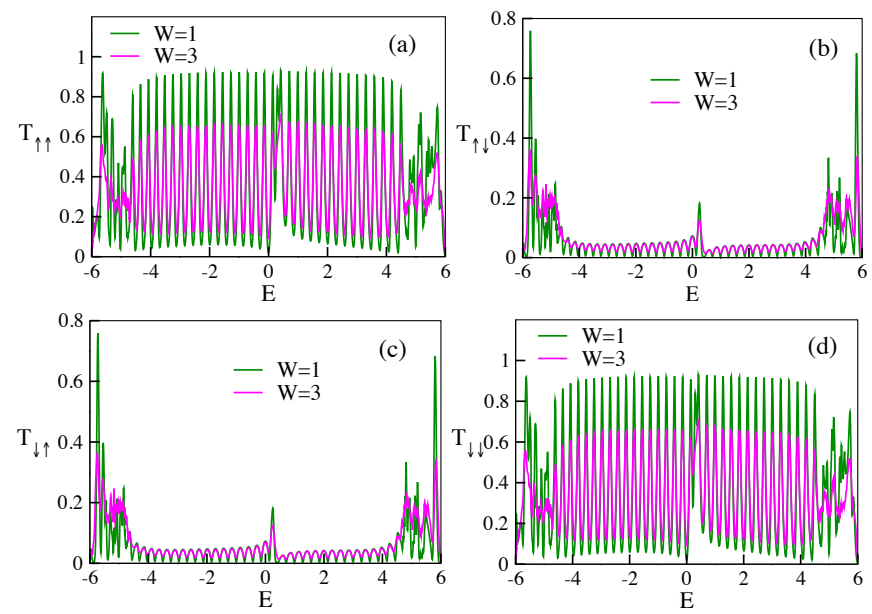

FIG. 12. (Color online) Behavior of Transmission probabilities (a) $T_{\uparrow \uparrow}$, (b) $T_{\uparrow \downarrow}$, (c) $T_{\downarrow \uparrow}$ and (d) $T_{\downarrow \downarrow}$ are shown as a function of incoming electron energy $E$ for the same disordered ring as taken in Fig. 11 but with $\mathrm{AB}$ flux $\left(\Phi=\Phi_{0} / 2\right)$. The green and magenta colors represent the results for the disorder strength $W=1$ and $W=3$, respectively. Other conditions are kept same as in Fig. 9.

tral gap appearing in the spectra is robust to disorder. Gap-widths do not change with the increase of disorder strength.

Also, we check our result for $\Phi=\Phi_{0} / 2$ which is illustrated in Fig. 12. For this value of $\mathrm{AB}$ flux, transmission probabilities decrease similar to the case of $\Phi=\Phi_{0} / 4$. For $\Phi=\Phi_{0} / 2$, the gap disappears as in the case of absence of disorder.

Therefore, we can say that the zero-energy crossings and the zero-energy transmission peaks at $\Phi=0$ and half-flux quantum values are not robust to non-magnetic impurity as evident from the energy spectra as well as the transmission spectra of the helical ring in presence of RSOI.

At the end, we would like to emphasize that in order to study the effect of disorder on the transport properties of the helical ring we have chosen 2/3-rd sites of the ring as disordered. Our results are valid for other disorder 
configurations even in presence of single impurity. To visualize the prominent effect of disorder we incorporate more than single impurity as in our model we consider only a single propagating mode or channel ${ }^{69-71}$.

\section{SUMMARY AND CONCLUSIONS}

To summarize, we have explored the spin-dependent transmission phenomena in a $1 \mathrm{D}$ ring with long-range hopping. In order to introduce the time-reversal counter part, we have considered the signs of the hopping corresponding to the two opposite spins are different. The functional form of the hopping has allowed us to manage the periodicity very nicely. We have applied AB flux along the axis of the ring. Our model is described in tight-binding framework. Using Green's function technique, we have calculated the transmission probabilities of the electrons through the ring and show the possibility of getting finite spin polarization by tuning AB flux only. The polarization also depends on the energy of the incoming electron.

Two counter-propagating states with opposite spins called as helical states carry the current throughout the ring. These helical states are very much similar to the 1D edge states of a 2D topological insulator. They are characterized by linear dispersion relations. Similar crossings are also present in our helical ring model energy spectra. In topological insulator Rashba spin-orbit interaction plays an important role. Also, the helical edge states are immune to non-magnetic impurity. To verify whether the counter-propagating edge states obtained in our case are protected by topology or not we have investigated the electron transport properties in presence of Rashba spinorbit interaction and static disorder. The helical states obtained in our model are sensitive to individual as well as combined effects of RSOI and disorder. RSOI destroys the zero-energy state and a gap appears around $E=0$. Whereas, presence of static disorder within the system results in shifting of those zero-energy state as well as the reduction in magnitude of the zero-energy transmission peak. Indeed, they also get affected in presence of both RSOI and disorder. Therefore, we conclude that the two counter-propagating states of the ring do not mimic the topological insulator edge states as the zero-energy transmission peaks as well as zero-energy crossings in the spectra are not robust to static random disorder unlike the edge states of topological insulator.

Finally, we have done a model calculation with some parameter values. For example, we have taken the hopping parameter within the ring as $t=2 \mathrm{eV}$ for bare hopping integral and for Rashba $t_{r s o}=1.5 \mathrm{eV}$. Our result is valid as long as $t_{r s o}<t$. For this Rashba value we also do not need to worry about the Rashba decay length towards the inside of the ring (tangential direction). It will not affect our main result depending on the aspect ratio (width/radius) of the ring ${ }^{72}$. We have chosen $\tau$ in order to study the strong coupling regime between the ring and the leads. One can also concentrate on the weakcoupling regime for exploring the transmission probability. In that case, our main result will remain invariant, only the peak widths will change. With the change of the other parameter values say $t$ or $t_{0}$ our results will change quantitatively keeping the qualitative nature consistent.

As far as the practical realization of our model is concerned, a quantum ring may be fabricated at the interface of two semiconducting materials possessing significant RSOI. For instance, in InAs semiconductor, the Rashba parameter $\alpha \sim 2 \times 10^{-11} \mathrm{eV} \mathrm{m}^{73}$. The strength of hopping due to Rashba is related to the Rashba parameter as, $\alpha /(2 \delta)$ where $\delta$ is the lattice constant ${ }^{62}$. If we take $\delta \sim 5 \mathrm{~nm}$ then $t_{r s o} \sim 0.2 \mathrm{eV}$ whereas for the above-mentioned lattice constant the nearest neighbor hopping integral is $\sim 2 \mathrm{eV}^{74}$. The magnitude of the external magnetic field can be $B \sim 3.2 \mathrm{mT}$ for a ring of radius $r \sim 0.2 \mu \mathrm{m}^{62}$. For our analysis, we have taken the parameter values particularly $t_{r s o}$ higher in magnitude. However for all other values of Rashba hopping strength, the quantitative values of our results will change keeping the qualitative nature unchanged as long as $t_{r s o}<t$.

\section{ACKNOWLEDGMENTS}

PD would like to acknowledge Shumpei Masuda, Florian Gebhard and Diptiman Sen for useful discussions and comments. PD thanks Science and Engineering Research Board (SERB), Department of Science and Technology (DST), India for the partial financial support through National Post-Doctoral Fellowship (File No. PDF/2016/001178). AMJ also thanks DST, India for financial support. Finally, we would like to acknowledge Indian citizens for supporting research in basic science.

\section{Appendix: Analytical treatment of the dispersion relation with the $A B$ flux}

The energy dispersion relation for the helical ring can be obtained analytically as follows.

Let us first consider the situation where there is no AB flux and Rashba spin-orbit interaction. For this simplest case we have no off-diagonal term in the hopping matrix. We can decouple matrix for the two spin states and derive the relation for each spin of the electron separately. The long-range hopping between $m$-th and $n$-th site in absence of $\mathrm{AB}$ flux and Rashba spin-orbit interaction is given by (for a particular $\sigma$ say +1 ),

$$
t_{m, n}=i t(-1)^{m-n} \frac{1}{(N / \pi) \sin [\pi(m-n) / N]}
$$

Now, we start from the Schrödinger equation and obtain 
the following difference equation,

$$
E \psi_{n}=-i t \sum_{m \neq n}(-1)^{m-n} \frac{1}{(N / \pi) \sin [\pi(m-n) / N]} \psi_{m}
$$

As the ring is periodic, we take Bloch wave solution $\left(\psi_{m} \sim e^{i k m a}\right)$ and get

$$
E=i t \sum_{m \neq n}(-1)^{m-n} \frac{1}{(N / \pi) \sin [\pi(m-n) / N]} e^{i k(m-n) a}
$$

Applying the periodic boundary condition, we have

$$
k=\frac{2 \pi l}{N a}
$$

where $l$ is an integer and it runs within the range

$$
-\frac{(N-1)}{2} \leq l<\frac{(N-1)}{2}
$$

Simplifying Eq. (A.3) we get the dispersion relation as,

$$
E=t k a
$$

Similarly we can find the $E-k$ relation for the other spin state $\sigma=-1$ for which we get a similar equation with a negative sign. Therefore, for any $\sigma$ it is given by,

$$
E=\sigma t k a
$$

where $\sigma= \pm 1$. This linear dispersion relation was primarily obtained Refs. 43 and 44 . It is completely in contrast to that obtained in ordinary ring with nearestneighbor hopping where we get cosine function in the dispersion relation ${ }^{13}$. The linear function is discontinuous at the Brillouin zone boundary keeping the periodicity of $2 \pi / a$. This allows us to get rid of the Fermion doubling problem which generally arises in other discrete models of helical edge states having continuous dispersion relation $^{13}$. For continuous function we have two end points of the Brillouin zone that are identical to each other.

This linear dispersion relation of the helical ring also holds in presence of magnetic field. In presence of $\mathrm{AB}$ flux we have,

$$
E=\sigma t\left(k a+\frac{2 \pi \Phi}{N \Phi_{0}}\right)
$$

Here, a question may arise. The energy spectra we have shown in this manuscript is more complicated instead of linear behavior. Our numerical spectra exactly match with that obtained by analytical calculation without any simplification. Without simplification we have the energy dispersion relation as,

$$
E=\sum_{m=1}^{(N-1) / 2}(-1)^{m} \frac{2 t \sin \left[m\left(k a+\frac{2 \pi \Phi}{N \Phi_{0}}\right)\right]}{(N / \pi) \sin (m \pi / N)} .
$$

Additionally, in presence of Rashba spin-orbit interaction we have additional off-diagonal terms in the hopping matrices. We have matrix form of the difference equation,

$$
E \boldsymbol{I} \psi_{m}=\sum_{n}\left(\begin{array}{cc}
t_{m, n}^{l} & -i t_{r s o} e^{-i \phi_{m, n}} \\
i t_{r s o} e^{i \phi_{m, n}} & -t_{m, n}^{l}
\end{array}\right) \psi_{n}
$$

Now again we assume the Bloch wave form for the wave function and solve it. Finally we arrive at the expression,

$$
E= \pm \sqrt{\xi(k)^{2}+\xi^{\prime}(k)^{2}} .
$$

where the form of $\xi(k)$ is exactly same as written in the right hand side of Eq. (A.9) and that for $\xi^{\prime}(k)$ is given by,

$$
\xi^{\prime}(k)=2 t_{r s o} \sum_{m=1}^{(N-1) / 2} \sin \left[m\left(\pi+k a-\frac{2 \pi \Phi}{N \Phi_{0}}\right)\right] .
$$

We show Eq. (A.11) which is exactly equivalent to the numerical one.
* paramitad@iopb.res.in

$\dagger$ arijit@iopb.res.in

‡ jayan@iopb.res.in

1 S. Wolf, D. Awschalom, R. Buhrman, J. Daughton, S. Von Molnar, M. Roukes, A. Y. Chtchelkanova, and D. Treger, Science 294, 1488 (2001).

2 I. Žutić, J. Fabian, and S. D. Sarma, Rev. Mod. Phys. 76, 323 (2004).

3 D. Frustaglia, M. Hentschel, and K. Richter, Phys. Rev. Lett. 87, 256602 (2001).

4 T. Koga, J. Nitta, H. Takayanagi, and S. Datta, Phys. Rev. Lett. 88, 126601 (2002).
${ }^{5}$ H.-F. Lü and Y. Guo, Appl. Phys. Lett. 91, 092128 (2007).

${ }^{6}$ Y. A. Bychkov and E. I. Rashba, J. Phys. C: Solid State Phys. 17, 6039 (1984).

7 D. Bercioux and P. Lucignano, Rep. Prog. Phys. 78, 106001 (2015).

8 J. C. Egues, G. Burkard, and D. Loss, Phys. Rev. Lett. 89, 176401 (2002).

9 J. Nitta, T. Akazaki, H. Takayanagi, and T. Enoki, Phys. Rev. Lett. 78, 1335 (1997).

10 M. Wang and K. Chang, Phys. Rev. B 77, 125330 (2008).

11 H.-F. Cheung, Y. Gefen, and E. K. Riedel, IBM J. Res. Dev. 32, 359 (1988). 
12 M. Büttiker, Y. Imry, and M. Y. Azbel, Phys. Rev. A 30, 1982 (1984).

13 H.-F. Cheung, Y. Gefen, E. K. Riedel, and W.-H. Shih, Phys. Rev. B 37, 6050 (1988).

14 H. F. Cheung, E. K. Riedel, and Y. Gefen, Phys. Rev. Lett. 62, 587 (1989).

15 P. S. Deo and A. Jayannavar, Phys. Rev. B 50, 11629 (1994).

16 A. Jayannavar and P. S. Deo, Phys. Rev. B 49, 13685 (1994).

17 A. Jayannavar and P. S. Deo, Phys. Rev. B 51, 10175 (1995).

18 R. Deblock, R. Bel, B. Reulet, H. Bouchiat, and D. Mailly, Phys. Rev. Lett. 89, 206803 (2002).

19 J. M. McLellan, M. Geissler, and Y. Xia, J. Am. Chem. Soc. 126, 10830 (2004).

20 F. Sun, J. C. Yu, and X. Wang, Chem. Mater. 18, 3774 (2006).

21 A. Yacoby, R. Schuster, and M. Heiblum, Phys. Rev. B. 53, 9583 (1996).

22 A. C. Bleszynski-Jayich, W. E. Shanks, B. Peaudecerf, E. Ginossar, F. von Oppen, L. Glazman, and J. G. E. Harris, Science 326, 272 (2009).

23 Y. Aharonov and D. Bohm, Phys. Rev. 115, 485 (1959).

${ }^{24}$ V. Moldoveanu and B. Tanatar, Phys. Rev. B 81, 035326 (2010).

25 J. Maciejko, E.-A. Kim, and X.-L. Qi, Phys. Rev. B 82, 195409 (2010).

26 A. Rod, T. L. Schmidt, and S. Rachel, Phys. Rev. B 91, 245112 (2015).

27 Z.-F. Jiang and W.-Y. Shan, arXiv:0908.0890 (2009).

28 M. Z. Hasan and C. L. Kane, Rev. Mod. Phys. 82, 3045 (2010).

29 X. L. Qi and S. C. Zhang, Rev. Mod. Phys. 83, 1057 (2011).

30 C. L. Kane and E. J. Mele, Phys. Rev. Lett. 95, 226801 (2005).

31 X.-L. Qi and S.-C. Zhang, Phys. Today 63, 33 (2010).

32 B. A. Bernevig, T. L. Hughes, and S.-C. Zhang, Science 314, 1757 (2006).

33 M. König, S. Wiedmann, C. Brüne, A. Roth, H. Buhmann, L. W. Molenkamp, X.-L. Qi, and S.-C. Zhang, Science 318, 766 (2007).

${ }^{34}$ D. Culcer, E. Hwang, T. D. Stanescu, and S. D. Sarma, Phys. Rev. B 82, 155457 (2010).

35 K. Sun, Z. Gu, H. Katsura, and S. D. Sarma, Phys. Rev. Lett. 106, 236803 (2011).

36 A. Ström, H. Johannesson, and G. Japaridze, Phys. Rev. Lett. 104, 256804 (2010).

37 A. Ström, H. Johannesson, and P. Recher, Phys. Rev. B 91, 245406 (2015).

38 J. Moore, Nature Phys. 5, 378 (2009).

39 T. Zhang, P. Cheng, X. Chen, J.-F. Jia, X. Ma, K. He, L. Wang, H. Zhang, X. Dai, Z. Fang, et al., Phys. Rev. Lett. 103, 266803 (2009).

40 C. L. Kane and E. J. Mele, Phys. Rev. Lett. 95, 146802 (2005).

41 H. Jiang, L. Wang, Q.-f. Sun, and X. Xie, Phys. Rev. B 80, 165316 (2009).

42 H. B. Nielsen and M. Ninomiya, Phys. Lett. B 105, 219 (1981).
43 S. Masuda and Y. Kuramoto, Phys. Rev. B 85, 195327 (2012).

44 F. Gebhard and A. E. Ruckenstein, Phys. Rev. Lett. 68, 244 (1992).

45 P. Jurcevic, B. P. Lanyon, P. Hauke, C. Hempel, P. Zoller, R. Blatt, and C. F. Roos, arXiv:1401.5387 (2014).

46 P. Richerme, Z.-X. Gong, A. Lee, C. Senko, J. Smith, M. Foss-Feig, S. Michalakis, A. V. Gorshkov, and C. Monroe, Nature 511, 198 (2014).

47 H. Cheraghchi, S. Fazeli, and K. Esfarjani, Phys. Rev. B 72, 174207 (2005).

48 G.-P. Zhang and S.-J. Xiong, Euro. Phys. J. B 29, 491 (2002).

49 S.-J. Xiong and G.-P. Zhang, Phys. Rev. B 68, 174201 (2003).

50 R. F. Loring, H. C. Andersen, and M. Fayer, J. Chem. Phys. 80, 5731 (1984).

51 A. Della Giusta and G. Ottonello, Phys. Chem. Min. 20, 228 (1993).

52 F. A. de Moura and M. L. Lyra, Phys. Rev. Lett. 81, 3735 (1998).

53 L. Levitov, Ann. Phys.(Leipzig) 8, 507 (1999).

54 H. Yamada, Int. J. Mod. Phys. B 18, 1697 (2004).

55 B. Giese, Acc. Chem. Res. 33, 631 (2000).

${ }_{57}$ G. B. Schuster, Acc. Chem. Res. 33, 253 (2000).

57 G. Celardo, R. Kaiser, and F. Borgonovi, arXiv:1604.07868 (2016).

58 H. Peng, K. Lai, D. Kong, S. Meister, Y. Chen, X.-L. Qi, S.-C. Zhang, Z.-X. Shen, and Y. Cui, Nature Mater. 9, 225 (2010).

59 P. Michetti and P. Recher, Phys. Rev. B 83, 125420 (2011).

60 R.-L. Chu, J. Li, J. Jain, and S.-Q. Shen, Phys. Rev. B 80, 081102 (2009).

61 S. Datta, Electronic transport in mesoscopic systems (Cambridge university press, 1997).

62 C. P. Moca and D. C. Marinescu, J. Phys.: Condens. Matter 18, 127 (2005).

63 L. Sheng, D. Sheng, and C. Ting, Phys. Rev. Lett. 94, 016602 (2005).

${ }^{64}$ R. Peierls, Z. Phys. 80, 763 (1933).

65 P. Dutta, S. K. Maiti, and S. N. Karmakar, Euro. Phys. J. B 85, 1 (2012).

66 P. W. Anderson, Phys. Rev. 109, 1492 (1958).

67 C. Soukoulis and E. Economou, Waves in Random Media 9, 255 (1999).

68 D. Bercioux, M. Governale, V. Cataudella, and V. M. Ramaglia, Phys. Rev. Lett. 93, 056802 (2004).

69 P. S. Deo and A. Jayannavar, Mod. Phys. Lett. B 7, 1045 (1993).

70 S. S. Chowdhury, P. S. Deo, A. K. Roy, and M. Manninen, New J. Phys. 10, 083014 (2008).

71 S. Bandopadhyay, R. Krishnan, and A. Jayannavar, Solid State Communs. 131, 447 (2004).

72 D. Frustaglia and K. Richter, Phys. Rev. B 69, 235310 (2004).

73 V. Mourik, K. Zuo, S. M. Frolov, S. Plissard, E. Bakkers, and L. Kouwenhoven, Science 336, 1003 (2012).

74 F. Mireles and G. Kirczenow, Phys. Rev. B 64, 024426 (2001). 